\title{
Neutral atom transport from the termination shock to $1 \mathrm{AU}$
}

\author{
Maciej Bzowski* and Sławomir Tarnopolski* \\ ${ }^{*}$ Space Research Centre PAS, Bartycka 18A, 00-716 Warsaw, Poland
}

\begin{abstract}
Dynamics of H, D, and heavy Energetic Neutral Atoms (ENA) between the termination shock and $1 \mathrm{AU}$ is discussed in the context of the forthcoming NASA SMEX mission IBEX. In particular, effects of the velocity-dependent radiation pressure on atomic trajectories are considered and ionization losses between TS and $1 \mathrm{AU}$ are studied. It is shown, among others, that most of the dynamical effects and ionization losses are induced within a few AU from the Sun, which translates to the time domain into $\sim 1-3$ solar rotations before detection. This loosens considerably time requirements for tracking the ionization and radiation pressure history to just prior 3 months. ENA seem excellent tracers of the processes within the heliospheric interface, with the transport effects between the termination shock and detector relatively mild and easy to account for.
\end{abstract}

\section{INTRODUCTION}

This article is a part of reconnaissance for a NASA SMEX mission IBEX ([1]; McComas et al, this volume). IBEX will be a spin-stabilized Earth satellite observing Energetic Neutral Atoms (ENA) in the $0.01-6 \mathrm{keV}$ energy band, with sensors looking perpendicularly to the spin axis directed at the Sun. The prime target of the mission will be imaging of the heliospheric interface via neutral atoms and the goal of an individual observation will be to obtain the kinematic parameters of the locally registered atom beyond the termination shock (TS). To that end, understanding the processes affecting the atoms between their source region and the detector is needed. In particular, a control on the modifications of the kinematic parameters of the atoms between the source region and the detector and a grasp on loss processes underway is necessary.

Since the ENA gas inside TS is collisionless at all energies, then - from Boltzmann equation - the transport of ENA from TS to the inner heliosphere is governed solely by dynamic effects and gain and loss processes.

The losses include all known ionization processes operating in the heliosphere: charge exchange, EUV ionization, and electron impact [2]. For inward-traveling ENA, gains (considered by IBEX as unwanted background) include charge exchange between CME, CIR and pickup ions and all kinds of neutral populations present inside TS, as well as production of ENA in the plasma environment of the spacecraft (McComas et al., this volume) and will be neglected. 

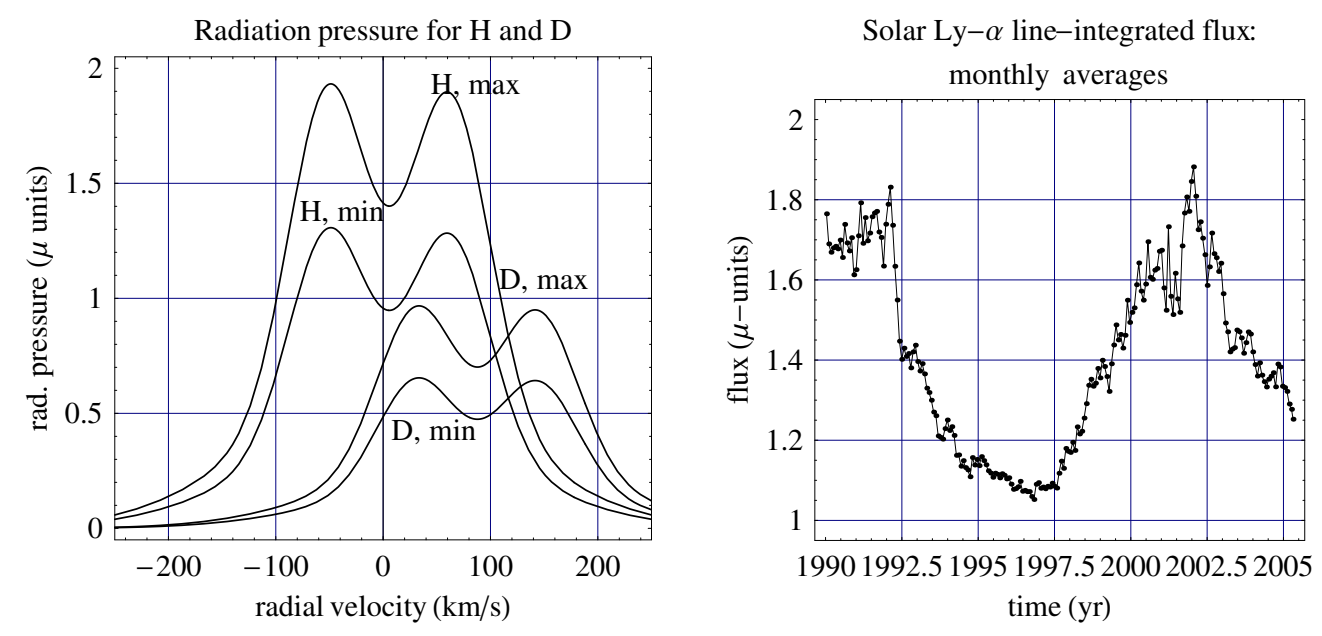

FIGURE 1. Radiation pressure force acting on $\mathrm{H}$ and $\mathrm{D}$ atoms as function of atomic radial velocity for solar minimum and maximum conditions, expressed in the units of solar gravity force ("the $\mu$ units") and the net line-integrated solar Lyman- $\alpha$ flux for the past $\sim 1.5$ solar cycle, also expressed in the $\mu$ units.

\section{DYNAMICS OF H AND D ATOMS WITH DOPPLER EFFECT}

From dynamical point of view, heliospheric ENA belong to two categories: sensitive and insensitive to solar radiation pressure. The sensitive ones include only hydrogen the most abundant heliospheric element - and its isotope deuterium. All atoms are, of course, sensitive to solar gravity.

All neutral atoms in the heliosphere obey the equation of motion in the form:

$$
d^{2} \vec{r} / d t^{2}=-G M\left(1-\mu\left(v_{r}, I(t)\right)\right) \vec{r} /|\vec{r}|^{3}
$$

where $v_{r}=(d \vec{r} / d t) \cdot \vec{r} /|\vec{r}|$ is radial velocity and $G M$ the gravity constant times solar mass. $I(t)$ is the line-integrated solar Lyman- $\alpha$ flux. For all atoms heavier than D the radiation pressure factor $\mu \equiv 0$ and the equation of motion reduces to the simple Keplerian case, with the ideal hyperbolic solution.

The expected range of velocities of heliospheric $\mathrm{H}$ and D ENA is $\sim 50-1000 \mathrm{~km} / \mathrm{s}$. Beacuse of the geometry of the lines of sight, IBEX is interested only in the inwardtraveling atoms, with velocities in the source region pointing so that at $1 \mathrm{AU}$ they will be at perihelion. Such atoms are being detected at 0 radial velocity with respect to the Sun, but cover most of their paths with radial velocities almost equal to their net velocities. Consequently, if their net velocity is larger than the spectral range of the solar Lyman- $\alpha$ line, the atoms do not experience any radiation pressure at all all the way except within a few AU from the Sun, where before detection they reduce their radial velocities to 0 . Since, however, they are fast, their trajectories will be weakly modified by solar gravity and hence close to straight lines. On the other hand, slower ENA (whose velocities remain within the spectral range of the solar line), will be affected by radiation pressure, which acts against solar gravity. Consequently, they also will follow trajectories close to straight lines, except perhaps within a few AU from the detector. 
The behavior of solar Lyman- $\alpha$ line from solar minimum to maximum has been recently surveyed by [3]. Based on these data it was possible to fit a general formula (Tarnopolski, thesis, in preparation):

$$
\mu\left(v_{r}, I\right)=(a+b I)\left(\exp \left[-c v_{r}^{2}\right]\left(d+f \exp \left[-g v_{r}-h v_{r}^{2}\right]+j \exp \left[k v_{r}-m v_{r}^{2}\right]\right)\right)
$$

which describes the radiation pressure of $\mathrm{H}$ or $\mathrm{D}$ atoms traveling at radial velocity $v_{r}$ with respect to the Sun. The formula, shown for $\mathrm{H}$ and $\mathrm{D}$ and solar minimum and maximum conditions in the left-hand panel of Fig 1, is parametrized by the sole parameter $I$, which is the line-integrated solar Lyman- $\alpha$ flux, whose variations during the solar cycle (SOLAR 2000, [4]) are shown in the right-hand panel of Fig 1].

Due to isotope effect, the deuterium resonant Lyman- $\alpha$ frequency is shifted blueward by $\sim 80 \mathrm{~km} / \mathrm{s}$ with respect to $\mathrm{H}$. Hence the $\mathrm{D}$ atoms are sensitive to a different part of the solar Lyman- $\alpha$ profile than $\mathrm{H}$ atoms and due to the mass difference the radiation pressure potentially acting on $\mathrm{D}$ atoms is additionally reduced two-fold with respect to $H$. The two effects combined will yield different dynamics of the $H$ and D ENA: while radiation pressure for $\mathrm{H}$ is approximately symmetric with respect to 0 radial velocity, this is not the case for $\mathrm{D}$ and the effective radiation pressure acting on the incoming $\mathrm{D}$ atom will be much lower than on a $\mathrm{H}$ atom launched from the termination shock with the same speed (see Fig 1, left-hand panel). Consequently, the dynamic behavior of the incoming D ENA is expected to be more similar to the behavior of purely-Keplerian heavy atoms than to that of $\mathrm{H}$ atoms.

Results presented in the following part of the paper are based on numerical solutions of Equ.(1) with the $\mu$ factor taken from Equ.(2), evaluated for two fixed values of $I(t)$, appropriate for solar minimum and solar maximum. For heavy atoms the $\mu$ factor was set to 0 . The atoms were tracked from their perihelions at 1 AU to TS, with velocities at perihelion ranging from 50 to $400 \mathrm{~km} / \mathrm{s}$. Along with the trajectory tracking the probability of ionization for an assumed ionization rate at $1 \mathrm{AU}$ was calculated.

\section{RESULTS}

\section{Trajectories and energy}

All heliospheric ENA except those from the low end of the IBEX energy band follow almost straight line trajectories, with very little velocity change between TS and the detector at 1 AU. For detection speeds $v>\sim 150 \mathrm{~km} / \mathrm{s}$ there is practically no velocity change between TS and 1 AU regardless of the species and solar activity phase (Fig,2). For $v$ lower than this value, $\mathrm{H}$ atoms detected at velocities $v$ have TS launch velocities $v_{T S, H}>v_{T S, D / O}$, the launch velocities of the heavier species detected at the same $v$. Also, usually $v<v_{T S, H}$, while opposite is true for $v_{T S, D / O}$. Consequently, mean velocity of the $\mathrm{H}$ atoms detected at $v$ is higher than the mean velocities of the heavies and their travel time is shorter. For example, an $\mathrm{O}$ atom detected at $50 \mathrm{~km} / \mathrm{s}$ needs $\sim 22 \mathrm{yr}$ to arrive at $1 \mathrm{AU}$ from the crosswind TS, where it was launched at $\sim 26 \mathrm{~km} / \mathrm{s}$, while a $\mathrm{H}$ atom detected at the same $50 \mathrm{~km} / \mathrm{s}$ needs only $\sim 12 \mathrm{yr}$ to make the trip and it was launched at $\sim 60 \mathrm{~km} / \mathrm{s}$. During solar minimum, even the slowest H ENA travel almost 

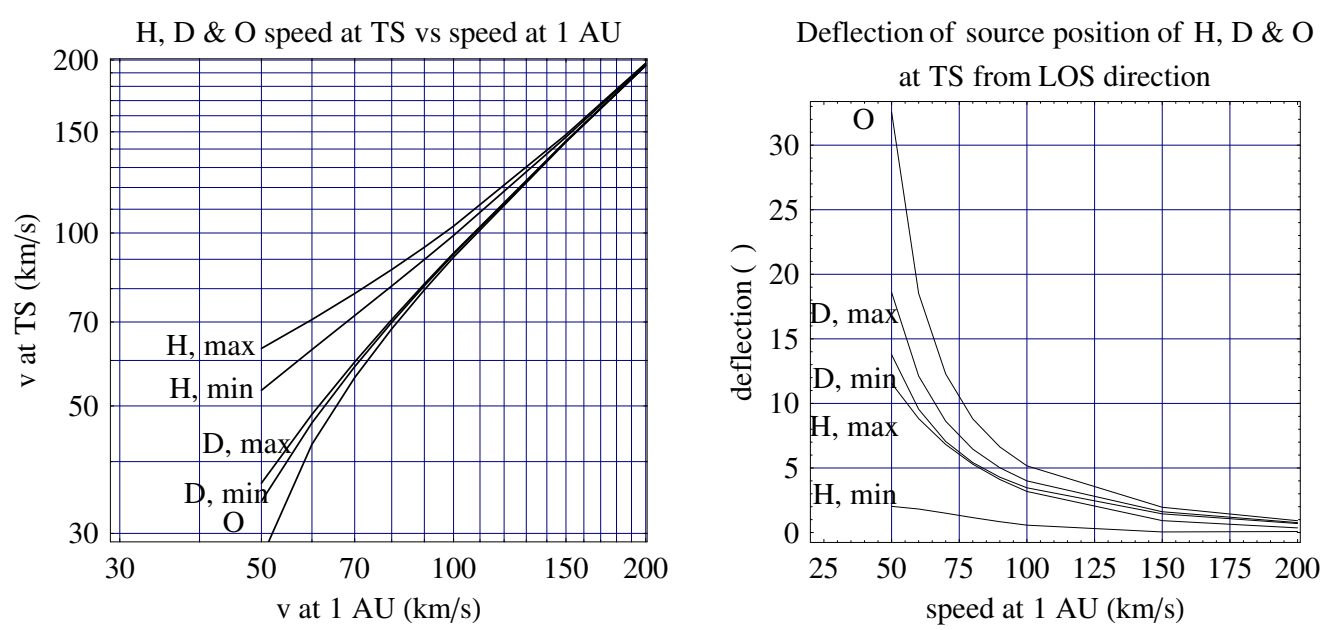

FIGURE 2. Change of speed of $\mathrm{H}, \mathrm{D}, \mathrm{O}$ atoms at solar minimum and maximum conditions between the termination shock and $1 \mathrm{AU}$ (left-hand panel) and angular separation between the line of sight (arrival direction at perihelion) and the source position at the termination shock for $\mathrm{H}, \mathrm{D}, \mathrm{O}$ atoms at solar minimum and maximum (right-hand panel). The results for $\mathrm{O}$ are valid for all heavy atoms.

constant speed, following straight lines in the whole IBEX energy range. This facilitates assessment of the travel times $t_{T S}$ from TS to $1 \mathrm{AU}$ for given detection speed $v$, which can be with good approximation calculated as:

$$
t_{T S}=r_{T S}(\theta) / v=125.74 /(1+0.253 \cos \theta) / v
$$

where $\theta$ is offset angle from the nose of TS and for $t_{T S}$ in yr $v$ must be in AU/yr. For the lowest-energy atoms $(\sim 100 \mathrm{~km} / \mathrm{s})$ the difference in travel time from the nose and tail of TS will be about $5 \mathrm{yr}$, but for typical H ENA at $400 \mathrm{~km} / \mathrm{s}$ it will be only 1 year. The shape of the termination shock was taken from Grzedzielski et al. (this volume).

Mapping of the launch position of $\mathrm{H}$ and $\mathrm{D}$ atoms at TS for a given detector pointing is practically 1 to 1 , with the deflection angle at the lower end of the energy band within $\sim 10^{\circ}$ (Fig 2, right-hand panel). Heavy atoms (insensitive to radiation pressure) are also weakly deflected except the slowest ones, which are deflected up to $\sim 30^{\circ}$ at $50 \mathrm{~km} / \mathrm{s}$. Deflection of all species drops below $5^{\circ}$ for detection speed $100 \mathrm{~km} / \mathrm{s}$. This is favorable for IBEX since it facilitates linking the pointing of detectors to the source region at TS.

Also favorable is the relatively short range of the combined solar gravity and solar radiation pressure acting on $\mathrm{H}$ ENA. The effective "collision time" of H/D ENA with the Sun before detection is only $\sim 0.25 \mathrm{yr}=3$ solar rotations, so the highly fluctuating solar Lyman- $\alpha$ output will need to be tracked back no longer than this interval.

\section{Ionization}

The survival probability of ENA against ionization between TS and $1 \mathrm{AU}$ is a function of the launch and detection speeds and of the ionization rate (assumed here to vary with 

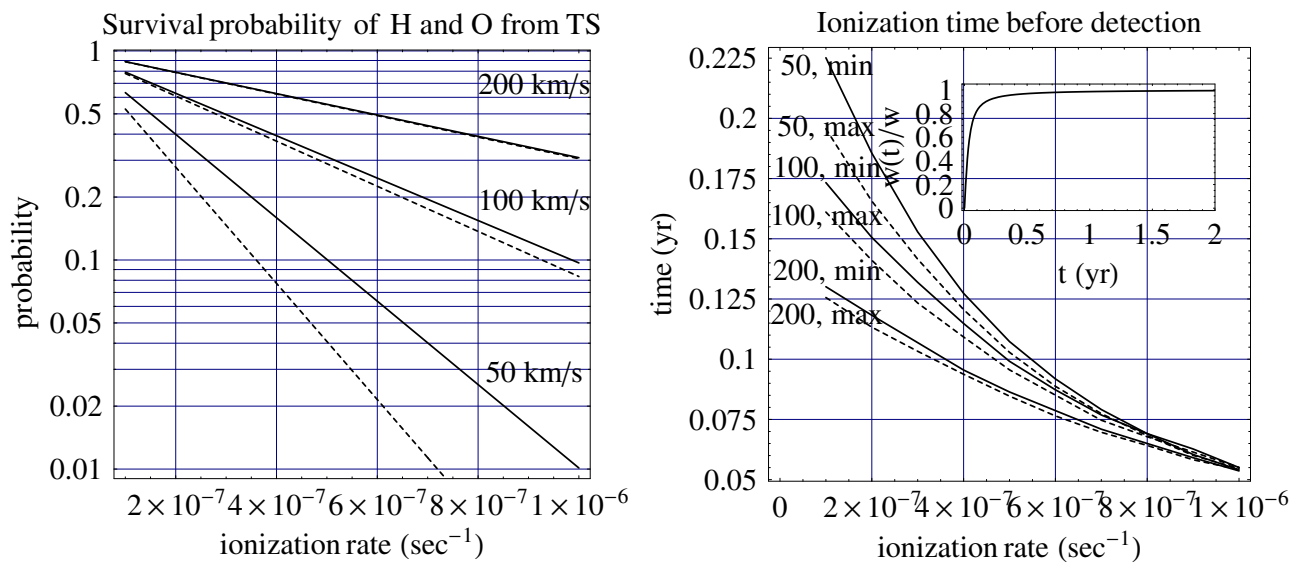

FIGURE 3. Left-hand panel: Survival probability of $\mathrm{H}$ (solid lines) and O ENA (broken lines) from TS to $1 \mathrm{AU}$ as function of the ionization rate at $1 \mathrm{AU}$ for various detection speeds. The solid lines in the left-hand panel correspond to H ENA, the broken lines to heavy atoms (like O); for $200 \mathrm{~km} / \mathrm{s}$ the two lines practically coincide. Right-hand panel: time of effective ionization defined in Equ. (4) for $\mathrm{H}$ at solar minimum and maximum conditions as function of the ionization rate at $1 \mathrm{AU}$ (right-hand panel) for various detection speeds, indicated in the figure, and for solar minimum (solid) and maximum conditions(broken). The inset in the right-hand panel shows an example of the course of relative survival probability $w_{r e l}(t)$ between TS and $r$.

heliocentric distance as $1 / r^{2}$ ). For the H ENA expected to be observed by IBEX it is quite large in the whole energy band except at the lowest end: for a $\mathrm{H}$ atom detected at $200 \mathrm{~km} / \mathrm{s}$ even for the highest expected ionization rate $10^{-6} \mathrm{~s}^{-1} 1 \mathrm{AU}$ [2] the probability is as much as $40 \%$, and for the lowest expected rate equal to $10^{-7} \mathrm{~s}^{-1}$ it is almost 1. For faster H, D, and heavy atoms the survival probability is correspondingly larger, depending on the ionization rate. On the other hand, the ionization losses will increase quickly with the decrease of detection speed, as shown in the left-hand panel of Fig 3 In the range of detection speeds shown, one expects some "radiation pressure bonus" in the survival probability for the $\mathrm{H}$ atoms (and to some extent for D) compared with the heavy atoms because of the larger mean speed of $\mathrm{H}$ atoms detected at a given $v$ in comparison with the mean speed of heavy atoms. This effect is significant only at the lowest end of the detection interval. For $\mathrm{H}$, some variations of the survival probability with the phase of the solar cycle are expected, but they are not big and probably will be masked by fluctuations of the actual ionization rate.

Most of the ionization losses occur shortly before detection. Denoting by $w_{1 \mathrm{AU}}$ the ionization probability of an atom underway from TS to $1 \mathrm{AU}$ and by $w(t)$ the probability of ionization at time $t$ before detection, we can define the relative survival probability $w_{\text {rel }}(t)$ and the ionization time $\tau_{\text {ion }}$ as:

$$
w_{\text {rel }}(t)=1-w(t) / w_{1 \mathrm{AU}} ; w_{\text {rel }}\left(\tau_{\text {ion }}\right) \equiv 0.8
$$

and determine the ionization times for different species and detection speeds. Results are shown in Fig 3 (right-hand panel). The ionization losses suffered by an atom underway 
from TS to $1 \mathrm{AU}$ are relatively small almost all the way down, and increase exponentially only within few months before detection. The exact value of $\tau_{i o n}$ is a function of detection speed and ionization rate, but generally one can say that the $80 \%$ ionization losses of all atoms occur within $0.1-0.25 \mathrm{yr}(\sim 1-3$ solar rotations $)$ before detection. The ionization times differ most for the lowest expected ionization rates and for the lowest energies at detection; for typical H ENA expected from the inner heliosheath the ionization time is expected at the level of one solar rotation period. This is favorable for IBEX operations because it means that interpretation of a signal requires monitoring of the local ionization rate only $\sim 1$ month backward in time.

\section{CONCLUSIONS}

The aspects of ENA transport from the termination shock to $1 \mathrm{AU}$ in the contexts of the planned IBEX mission can be summarized as follows.

Both heavy ENA and $\mathrm{H}$ and D ENA are excellent tracers of atomic parameters at and beyond TS. Velocity change between the termination shock and $1 \mathrm{AU}$ becomes noticeable only below $150 \mathrm{~km} / \mathrm{s}$ and deflection of the trajectories with respect to the launch site at TS beyond angular resolution of IBEX instruments for H ENA occurs only during solar maximum (which will be beyond IBEX nominal mission time) at the lowest limit of the energy band. Heavy atoms are a little stronger deflected, but the deflections exceed IBEX resolution only for detection speeds lower than $\sim 70 \mathrm{~km} / \mathrm{s}$.

Ionization losses between TS and 1 AU should not be a big problem for $\mathrm{H}$ ENA expected from the heliosheath. The survival probability drops down below $10 \%$ only for $\mathrm{H}$ ENA traveling at $1 \mathrm{AU}$ slower than $100 \mathrm{~km} / \mathrm{s}$. Most of the ionization losses will occur within a month or so before detection and hence should be relatively easy to take into account.

\section{ACKNOWLEDGMENTS}

This research was supported by the Polish MSRiT Grant 1P03D 00927.

\section{REFERENCES}

1. D. McComas, F. Allegrini, P. Bochsler, M. Bzowski, M. Collier, H. Fahr, H. Fichtner, P. Frisch, H. Funsten, S. Fuselier, G. Gloeckler, M. Gruntman, V. Izmodenov, P. Knappenberger, M. Lee, S. Livi, D. Mitchell, E. Möbius, T. Moore, D. Reisenfeld, E. Roelof, N. Schwadron, M. Wieser, M. Witte, P. Wurz, and G. Zank, "The Interstellar Boundary Explorer (IBEX)," in AIP Conf. Proc. 719: Physics of the Outer Heliosphere, 2004, pp. 162-181.

2. D. Ruciński, A. C. Cummings, G. Gloeckler, A. J. Lazarus, E. Möbius, and M. Witte, Space Sci. Res. 78, 73-84 (1996).

3. P. L. Lemaire, C. Emerich, J. C. Vial, W. Curdt, U. Schüle, and K. Wilhelm, "Variation of the full Sun hydrogen Lyman $\alpha$ and $\beta$ profiles with the activity cycle," in ESA SP-508: From Solar Min to Max: Half a Solar Cycle with SOHO, 2002, pp. 219-222. 
4. W. K. Tobiska, T. Woods, F. Eparvier, R. Viereck, L. Floyd, D. Bouwer, G. Rottman, and O. R. White, J. Atm. Terr. Phys. 62, 1233-1250 (2000). 